Relations industrielles

Industrial Relations

\title{
The Economics of Technological Change, by Nathan Rosenberg (Ed.), Middlesex, England, Penguin Modern Economics Readings, 1971, 509 pp.
}

\section{Jean Sexton}

Volume 28, numéro 1, 1973

URI : https://id.erudit.org/iderudit/028376ar

DOI : https://doi.org/10.7202/028376ar

Aller au sommaire du numéro

Éditeur(s)

Département des relations industrielles de l'Université Laval

ISSN

0034-379X (imprimé)

1703-8138 (numérique)

Découvrir la revue

Citer ce compte rendu

Sexton, J. (1973). Compte rendu de [The Economics of Technological Change, by Nathan Rosenberg (Ed.), Middlesex, England, Penguin Modern Economics Readings, 1971, 509 pp.] Relations industrielles / Industrial Relations, 28(1), 210-211. https://doi.org/10.7202/028376ar

Tous droits réservés @ C Département des relations industrielles de l'Université Laval, 1973
Ce document est protégé par la loi sur le droit d'auteur. L'utilisation des services d’Érudit (y compris la reproduction) est assujettie à sa politique d'utilisation que vous pouvez consulter en ligne.

https://apropos.erudit.org/fr/usagers/politique-dutilisation/ 
La préparation de cet ouvrage a exigé une somme de travail très considérable, puisque l'auteur et ses collaborateurs ont étudié toutes les décisions rendues par ces organismes après audition des parties, ce qui doit en faire tout de même au-delà d'une dizaine de milliers.

Les auteurs ont résumé et analysé des centaines de décisions qu'ils ont groupées par ordre de sujets. Chaque cas étudié contient un exposé des faits, l'objet du litige ainsi que la nature et les motifs de la décision rendue.

On peut facilement se rendre compte de l'utilité et des avantages de cette formule. D'une part, elle permet de réunir en un tout organique la jurisprudence qui s'est graduellement fixée au cours des années; d'autre part, elle peut aider ceux qui seraient intéressés à pousser la consultation plus loin que l'utilité pratique immédiate à tenter de reconstituer l'évolution de la législation du travail dans un domaine précis de l'application de ce droit et de mesurer les progrès réalisés au cours des vingt dernières années en passant de la Loi des relations ouvrières au Code du travail.

Il suffit, pour se rendre compte que la chose est ainsi rendue possible, de s'arrêter à quelques points déterminés. La notion de salarié en est un premier exemple. A travers des flottements toujours inévitables selon la pensée de ceux qui sont chargés d'interpréter la loi, on sent qu'il y a eu une tendance, sinon constante du moins réelle, vers un élargissement de la définition. Cela s'est fait sans doute par le texte de la loi luimême, mais également par le jeu des décisions. On ne peut s'empêcher de penser, par exemple aux mesureurs de bois, aux employés de l'Hydro-Québec, aux fonctionnaires de la Province, aux membres des professions libérales qui, il n'y a pas si longtemps, n'étaient pas considérés comme des salariés et, partant, ne bénéficiaient pas des avantages de la législation du travail. C'est ainsi que, graduellement, on en est venu à considérer que, pour être rangé parmi les non-salariés, il fallait que l'employé exerce une autorité réelle sur des subalternes, l'importance de sa fonction, si haute fût-elle, ne l'empêchant pas de se prévaloir des droits garantis aux travailleurs par le Code du travail.
Il ne faut pas oublier l'article $36 \mathrm{du}$ Code relatif à l'aliénation et à la con cession des entreprises. Introduite dans la loi au commencement de la décennie 1960 , cette disposition a donné lieu, au cours des dix années qui ont suivi, à une centaine de décisions importantes, quelques-unes d'entre elles ayant fait le sujet de débats fortement controversés et d'études approfondies.

On pourrait enfin parler des nombreuses causes se rapportant directement au droit d'accréditation lui-même qui y sont analysées.

Il suffit de feuilleter rapidernent cet ouvrage de quelque cinq cents pages pour en découvrir la valeur et l'utilité, cela d'autant plus que les périodiques spécialisés dans ce genre de publications n'avaient pu rendre compte que d'un nombre relativement restreint de décisions, les autres, certaines mêmes qui revêtaient une importance considérable, demeurant enfouies dans les dossiers.

On ne peut donc que souscrire à l'observation du juge Robert Sauvé qui écrit dans la préface de cet cuvrage : «On félicite d'habitude un auteur pour son travail. Ici, il faut vraiment faire plus. On doit lui rendre le témoignage d'avoir su faire un ouvrage de bénédictin....».

Gaston Descôteaux et ses collaborateurs ont abattu une tâche colossale et ont su la mener à bien.

André ROY

The Economics of Technological Change, by Nathan Rosenberg (Ed.), Middlesex, England, Penguin Modern Economics Readings, 1971, 509 pp.

Les changements technologiques ne constituent sûrement pas un sujet nouveau en sciences sociales en général et et relations industrielles plus particulièrement. Cependant, il reste encore à en connaître bien des aspects et surtout à trouver des solutions aux problèmes qu'ils suscitent. Ce livre attire l'attention sur une certaine façon, mais non la seule, de voir les choses dans ce domaine en soutenant que le raisonnement économique est un outil important pour la compréhension des causes et des conséquences du changement technologique. 
Ce « reading » est divisé en cinq parties principales et regroupe vingt-deux articles dont les dates de parution varient entre 1928 et 1966 avec une concentration dans la décennie 19551965. Une brève présentation précède chacune des parties et une bonne bibliographie propose aux lecteurs intéressés d'élargir leur intérêt.

Une première partie s'intéresse à la nature et au processus du changement technologique. Cette partie est teintée d'un intérêt historique par l'article de Schumpeter qui voit comme inévitablement relié l'instabilité de courte période du capitalisme et la croissance en longue période. Cette partie est complétée par les pensées de Usher, Ruttan et Blaug.

Puis, l'éditeur concentre son attention sur les facteurs déterminants du changement technologique. C'est ici qu'on tente de répondre à des questions telles le rôle des forces du marché sur le changement technologique, la réaction de la technologie aux pressions du marché et la distribution des bénéfices et des coûts dans le processus du changement technologique. Dans cette section, Schmookler, Nelson, Arrow et Fellner soulignent l'importance du profit comme motivateur d'activités innovatrices. Parker examine deux siècles de changements technologiques, Griliches rapporte un taux de rendement (privé et public) d'au-delà de $700 \%$ de certaines recherches en agriculture et Fellner examine l'impact sur les facteurs de production utilisés.

Dans un troisième temps, on offre quelques réflexions sur la diffusion de la nouvelle technologie. Fondamentalement, on soutient la thèse que les changements observés dans la distribution d'innovations particulières sont largement dus à des variables économiques telles le profit. Une série d'études de cas illustre ce point.

Dans une quatrième partie, on attaque les conséquences des changements technologiques sur la croissance économique. Des articles d'Abramovitz et de Solow tentent de quantifier cette contribution et concluent à la tendance généralisée de sur-estimation. Denison et Griliches présentent chacun leur version des choses. Il est regrettable que dans cette partie, comme dans tout le livre, on ne dise pas mot des conséquences des changements technologiques sur les travailleurs et les communautés. Il semble que ce soit un coût (privé et social) qui devrait préoccuper même l'économiste.

Finalement cet ouvrage présente quelques réflexions sur les aspects internationaux des changements technologiques en termes de concurrence internationale. Y a-t-il avantage à être un leader en ce domaine? Quelles influences peut-on noter sur les fonctions de production. jusqu'à quel point un pays peut-il absorber une technologie plus avancée?

Cet ouvrage présente sûrement un point de vue intéressant mais évidemment partiel sur les changements technologiques. C'est un ouvrage intéressant qui s'ajoute à la vaste littérature sur les changements technologiques.

Jean SEXTON

Perspectives on the Wage-Price-Unemployment Problem : Stop the Market, I Want to Get Off, Proceedings of the 1972 Conference of the Industrial Relations Management Association of British Columbia, February 1972, Edited by Vance F. Mitchell and W.T. Stanbury, Vancouver: Versatile $\mathrm{Pu}$ blishing, 1972, $178 \mathrm{pp}$.

Ce livre est le compte-rendu de la Huitième conférence annuelle de l'IRMA qui a eu lieu à Vancouver en février 1972 et qui portait sur le dilemme chômage-inflation. La liste des conférenciers comprenait Ronald Bodkin, Sylvia Ostry, Robert Flanagan, Donald Vial, John Young, Robert Bandeen, John Fryer, Abba Lerner et Eric Kierans.

Bodkin laisse entendre que, depuis 1965 , le dilemme chômage-inflation est devenu plus difficile au Canada, en ce sens que pour atteindre un taux donné de chồmage, il faut être prêt à accepter un taux d'inflation plus élevé d'environ $21 / 2 \%$ qu'auparavant. Il note que cette exacerbation du dilemme est due à la montée des anticipations inflationnistes, sans en expliquer cependant la source ultime. A son avis, ces anticipations ne dégénèreront pas en spirale irrésistible à long terme. Il lance aussi, avec Lerner, un slogan qui semble faire l'unanimité des économistes présents (sauf Young), à savoir que les coûts sociaux de l'infla- 